\title{
Téoros
}

Revue de recherche en tourisme

\section{La culture offerte en partage : un miroir à alouettes!}

\section{Pierre Mayrand}

Volume 2, numéro 2, juin 1983

Regards neufs sur les attractions touristiques du Québec

URI : https://id.erudit.org/iderudit/1080835ar

DOI : https://doi.org/10.7202/1080835ar

Aller au sommaire du numéro

Éditeur(s)

Université du Québec à Montréal

ISSN

0712-8657 (imprimé)

1923-2705 (numérique)

Découvrir la revue

Citer cet article

Mayrand, P. (1983). La culture offerte en partage : un miroir à alouettes!

Téoros, 2(2), 29-32. https://doi.org/10.7202/1080835ar d'utilisation que vous pouvez consulter en ligne.

https://apropos.erudit.org/fr/usagers/politique-dutilisation/ 


\title{
La culture offerte en partage: un miroir à alouettes!
}

\author{
Pierre Mayrand,
}

Le Québec foisonne d'une vie culturelle intense mais le sens de l'évolution de ses aspirations culturelles les plus authentiques échappe aux gens de l'extérieur, de même qu'il demeure incompris des sphères politiques. A peine une égratignure du cóté français ou des relations privilégiées ont crée un mythe qui a fait naître des besoins sur le plan touristique. Le visiteur américain, principal adepte du Québec, étant plutôt un voyageur en transit, ne semble pas avoir développé une véritable sensibilité a la différence québécoise. II ne voit, dans son voyage ici, que des poncifs et des avantages pratiques (proximité, confort, coüts,...). Hors Québec et Montréal, considéré comme des petits Paris à défaut du Paris réel, on est peu sensibilisé a autre chose qu'aux grands espaces et aux possibilités ludiques qu'ils renferment et à une vague excitation découlant d'un parler different.

La culture officielle québécoise, celle qu'on offre à partager avec des visiteurs, demeure largement déficitaire par rapport au potentiel existant et à la réalité. On offre donc un mirage, un miroir a alouettes.

"Pour le visiteur qui arrive aujourd'hui au Quebec, il n'est pas facile de detecter, du moins du premier regard, un ensemble cuiturel spécifique et vraiment original. Comment reconnaître la figure originale de cette culture? 111 Les touristes étant, le plus souvent, gens d'un epremier regard $n$, ils repartent avec ce qu'ont traduit pour eux les publicités officielles. Éloignés des sources vives d'où jaillit et se renouvelle la culture, les definisseurs d'image du secteur public comme de l'entreprise privée sont ignorants des véritables faits et enjeux culturels du Quebec d'aujourd'hui. Comme une majorité de la population dont ils ne font que véhiculer les hesitations, ils projettent une image imprécise, relevée de slogans réparateurs et répercuteurs, plutôtt qu'un sens convaincant du réel et de la sincérité.

La situation de la culture québécoise, en 1983, fait d'elle un produit difficile a construire selon les exigences connues de I'offre et de la demande touristiques 2 . Faut-il, pour autant, faire des mises-enboite, forcément factices, pour les visiteurs pressés ou tenter de les sensibiliser différemment? II $n^{\prime} y$ a pas de dilemme shakespearien sousjacent à cette question: une certaine uscénarisation w de notre savoir vivre et de notre savoir faire sur le plan culturel est possible - elle aiderait alors la qualité des rapports entre les visiteurs et les visités - mais elle doit venir des premiers concernés, c'est-à-dire des communautés de base où s'anime la vie culturelle du Québec. Cela est-il possible? Des expériences en cours ne laissent guère de doutes à ce sujet. Pour en comprendre le sens et la portée, il faut $d^{\prime}$ abord nous entendre sur les traits principaux de l'histoire de la culture québécoise et de l'état présent de son développement.

\section{Quelle culture québécoise?}

On nous présente culturellement comme des aparlants français». La langue nous différencie mais n'est pas nécessairement une culture. L'expression culturelle, au Québec, est très variée, selon les régions,

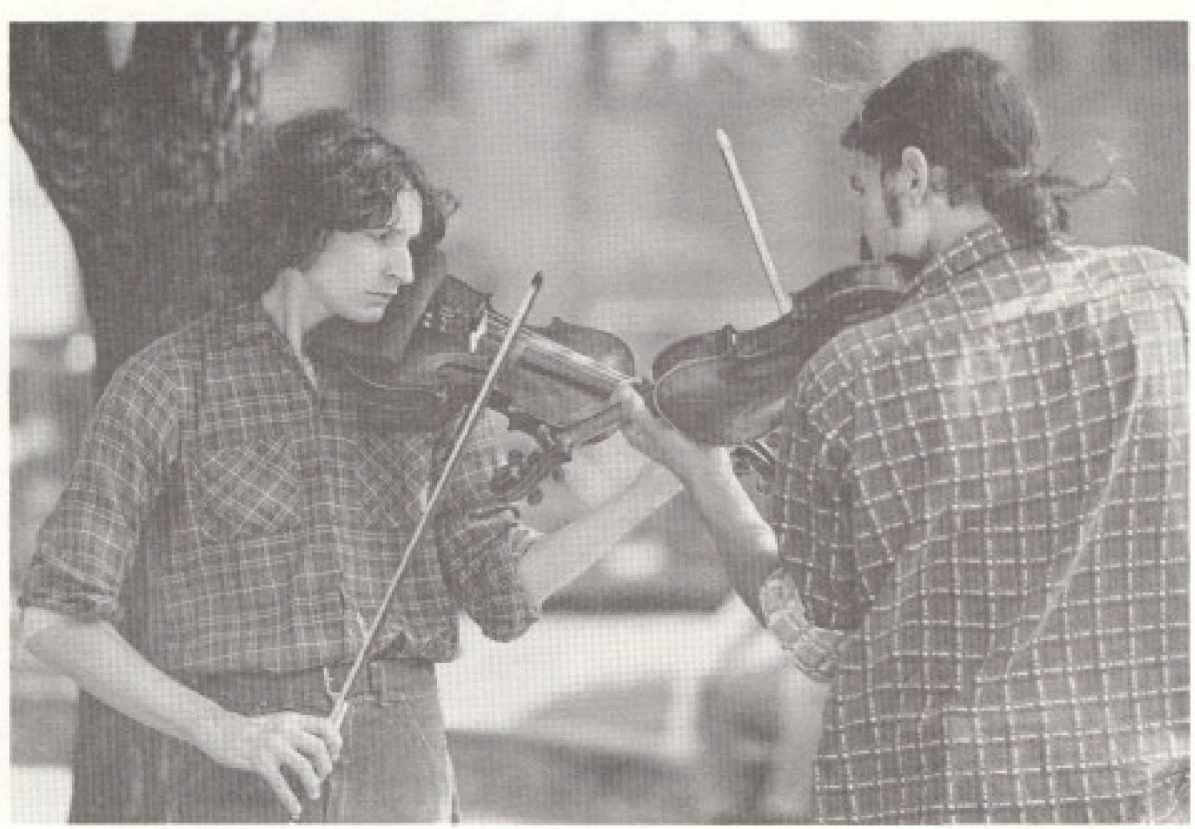

selon les milieux rural ou urbain, selon la particularité êthnique.

Comment présente-t-on les Québécois? Comme des gens differents, comme formant une mosaĭque? On peut fabriquer de toutes pièces, pour le tourisme, une culture québécoise, en cherchant un certain nombre de caractéristiques qui sont attrayantes, comme l'habillement colorế (les chemises a carreaux II. On cherche alors des choses qui pourraient se vérifier dans tous les cas, partout, qui formeraient alors comme des dénominateurs communs. On fabrique ces traits par un assemblage d'éléments, de produits culturels. Pour certains, la culture québécoise, c'est la ceinture fléchée; pour les élites de la culture nationale, c'est celle qui a passé la frontière (littérature, arts plastiques, chan= son, etc.).

Mais le Québécois privilégie toujours la culture vécue par rapport à la culture organisée. Les grandes productions, les grandes salles le laissent indifférent; il préferre les festivals, les fêtes, les manifestations populaires. $\alpha / / y$ a toujours, 
au Quebec et ailleurs, une culture officielle et une culture que l'on ne sait encore nommer.s.3) Les mours des Québecois, leurs habitudes de vie se traduisent surtout par le goût des rassemblements, des festivals. Autrefois, ces rassemblements se faisaient sur le parvis des eglises; aujourd'hui, c'est dans la rue. " Les grands événements de la vie, les noces par exemple, procuraient d'excellentes occasions de s'amuser en joyeuse compagnie. $w^{|4|}$ a La culture toute entiere est un

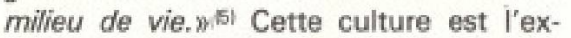
pression spontanée des gens; elle tire son origine de la cuisine, du salon, de la grange oû on se réunissait pour faire les activités saisonnières, cycliques. Aujourd'hui, le goût du rassemblement est exploité politiquement pour répondre aux besoins des Québécois de se réunir de temps à autre.

Face à ce trait culturell des Québécois à l'aise dans les expressions spontanées de la culture, d'une culture esauvagen, immédiate comme on la désigne parfois, comment se présente la culture organisée? Son premier caractère est de prétendre à I'universalité c'est-â-dire, dans le cas du Québec, de vouloir exprimer la culture unationales. C'est méconnaître un trait fondamental de la culture d'ici: sa diversité régionale.

Ce fait régional fait couler beaucoup d'encre. Sa réalité est difficile à saisir et pourtant, on s'en glorifie dans le discours tout en la niant dans les faits et dans les interventions publiques. «Dans un immense pays comme le nótre, et relativement peu peuple, les diversités régiona: les sont, plus que dans bien d'autres cas, sources d'originalité et de créativite. wit «En dehors de Montréal, le réseau d'établissements culturels lau sens restreint du mot) demeure neanmoins tres faible., (7) a'autres difficultés génent la mise en place d"une véritable politique du Joisir: elles sont liées a un phénomene tout a fait contemporain: /organisation du loisir. $x^{\left[{ }^{13}\right.}$

L'exemple du patrimaine est typique du décalage entre la culture organiśée et la culture vécue. On a un produit intéressant, révélateur de nos régions. Cela demande des équipements importants. Ceux qu'on a ne reflètent pas la réalité, les disparités régionales. Les musées ne sont pas planifiés dans l'espace, dans le temps ni dans leurs contenus; souvent ils ne sont pas reconnus dans leurs régions. Comme ils sont présentement, nos musées n'offrent pas de continuum sur le plan touristique. S'ils représentaient un scénario de la culture québécoise, cela pourrait devenir intéressant. Mais on ne peut pas demander à un touriste de parcourir tout un réseau de musées pour nous connaître et nous comprendre.
Guy Boulizon, dans son ouvrage intéressant sur les musées du Québec, en souligne la valeur: " $U n$ voyage surprenant, parfois deconcertant, le p/us souvent exa/tant... les museses publics ou prives sont de plus en plus, la "mémoire" vivante, populaire et bourgeoise, de ce pays.mpi L'auteur dit que dans les musées on découvre l'activité énorme des collectivités, les produits des individus et des groupes. Mais c'est tout ce qu'on y trouve; il $n^{\prime} y$ a aucune tentative pour aller plus loin, pour révéler la culture liée à ces activités et ces produits, pour la présenter aux visiteurs. ${ }^{101}$.

\section{L'action gouvernementale}

La culture organisée semble être l'affaire des organismes publics. Le Québec prépare un musée de la civilisation québécoise. Dans ce musée synthèse, il faudrait permettre aux régions de se réfléter. La recherche devrait se faire dans les régions d'abord pour ensuite alimenter le centre.
Des actions d'envergure comme celle-là s'entreprennent ici dans un contexte politique perturbé. Les grandes disparités sur le plan gouvernemental aboutissent à un manque de cohérence dans le produit culturel lui-même - les musées - et dans la façon de présenter le réseau aux visiteurs. Les deux paliers de gouvernement, sur ce terrain comme sur bien d'autres, travaillent à mettre de l'avant leurs concepts culturels respectifs - qui different grandement - et tout cela avec des moyens techniques divergents.

Le gouvernement fédéral se centre beaucoup plus sur les événements de la culture nationale qui soulignent l'unité, I'union des peuples alors que les Québécois sont axés sur la culture régionale. Le gouvernement d'Ottawa dispose de moyens techniques plus importants, ce qui lui permet d'assurer une permanence aux équipements. Ses réalisations ont l'air plus compétentes, plus professionnelles, mais

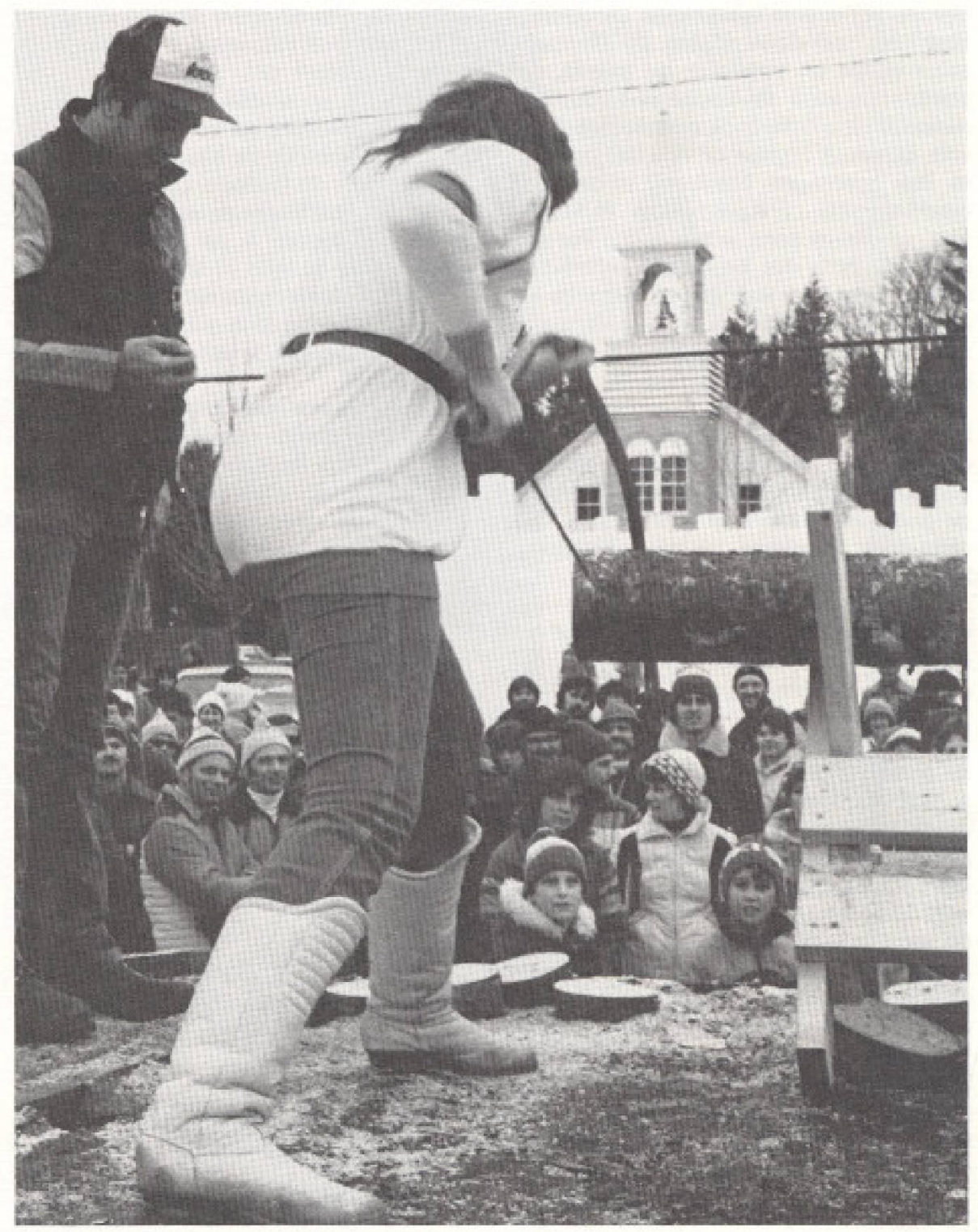

qDans un immense pays comme le nötre, les diversités régionales sont plus que dans bien d'autres cas sources d'originalité et de créativitế. Les festivals en sont le témoignage. 
il y manque une certaine chaleur. Chez les Québécois, l'approche est plus humaine, davantage reliée aux personnes; l'équipement, plus rattaché aux gens, ne peut pas êttre aussi permanent ni aussi stable.

Les structures sont donc imparfaites des deux côtés. De plus, les deux techniques d'animation ne se ressemblent pas du tout: le fédéral compte sur une information très poussée alors que le Québec mise sur des communications plus personnalisées. On peut observer cependant une certaine tendance a la normalisation par la création de centres d'interprétation du gouvernement du Québec. Les écomusées, produits originaux du Québec, ont à faire la synthèse de ces approches en résolvant le problème des ressources et des moyens.

Ces politiques divergentes se réflètent dans les stratégies de vente ou de promotion. Quand il s'agit d'ouvrir notre culture aux visiteurs, cela se fait toujours par le truchement des équipements, à travers ce qui est visible, observable. Orientés différemment et disposant de moyens inégaux. les produits offerts par le Québec en matière de patrimoine semblent moins sophistiqués et moins durables. Le visiteur ne sait rien de ces antagonismes intergouvernementaux: il ne peut qu'observer les différences visibles d'un musée à un autre, d'une attraction à une autre. Et les lieux qui apparaissent comme plus organisés rendent compte en même temps d'une culture wcanadiennen qui fait contraste avec les cultures locales des petits musées régionaux québécois, sympathiques certes, mais moins savamment présentés.

Le marketing finalement s'en ressent. La vallée du Richelieu par exemple, pourrait être un itinéraire intéressant pour découvrir le patrimoine québécois. Mais on a donné une fausse cohérence à cette zone. II n'y a pas d'équipernents pour mettre en valeur les sites; la relation entre la rivière et les montérégiennes n'est pas exploitée, il $n^{\prime} y$ a pas de signification, aucun système de relais, aucune unité entre les zones: on retrouve là une accumulation d'expériences disparates et un touriste n'y voit pas grand chose.

Autre exemple dans cette mểme région: le centre d'interprétation des patriotes a St-Denis devrait dépasser le fait historique, l'élargir, faire en même temps un rappel du vécu de ce temps, une histoire de l'environnement. Les rapports sont inexistants entre le centre d'interprétation du Mont St-Hilaire et le musée régional du Haut-Richelieu dans un corridor touristique majeur dont le potentiel demeure à peine exploité.

Au Québec, là encore, disparité êt incohérence du marketing. Le Québécois est mal organisé, ill ne sait pas rassembler. C'est pourquoi le marketing a été récupéré par des gens de l'extérieur qui fabriquent de toutes pièces notre image. Mais celle-ci ne ressemble pas à notre réalité. aLe sous-developpement économique, comme on le sait, designe moins linferiorite des performances par rapport a des standards établis qu'un ensemble de données structurelles qui fait qu'une collectivité $n$ 'a pas les moyens de prendre en mains et de développer ses potentialitess... Ce qui caracterise une société dite économique. ment sous-developpee, c'est le fait que ses priorités sont déterminées par d'autres... II en resulte le plus souvent des développements incomplets et sans harmonie., ${ }^{(11)}$

\section{L'expérience de la Haute-Beauce}

Le collectif des 13 villages de l'Ecomusée de la Haute-Beauce a entrepris a un moment donné une projection promotionnelle. II s'agissait d'une stratégie pour amener la population a se rapprocher de l'image projetée mais l'expérience de l'écomusée avait démontré qu'on pouvait atteindre cette image-là. L'image préalable se situait au niveau des concepts: ceux de région, de mouvement régional. La réalité du développement possible était connue: il fallait alors vendre l'idée à la population d'abord et aux visiteurs ensuite.

Le cas de la Haute-Beauce illustre le processus de développement culturel: il s'agit de permettre à une sous-région de $s^{\prime}$ affirmer comme produit original au sein de la région. Le processus permet de dégager et d'affirmer les caractéristiques régionales tant au profit du développement culturel de la population qu'à la qualité du produit offert à la connaissance du visiteur.

On a créé d'abord des conditions favorables pour que la population puisse obtenir les équipements dont elle a besoin pour valoriser sa région. Ces équipements se sont constitués en un réseau intégré, stable, attirant pour le touriste et lié a l'essor économique. Sur le plan touristique, cela donne un produit différent des autres et sa distinction est crébe par la population. Ce mouvement a commencé par la création d'infrastructures ce qui fut, pour la population, l'occasion de constater qu'elle pouvait se prendre en main.

L'attraction touristique doit être préparée de telle sorte que le visiteur s'y retrouve et retrouve le visité à travers elle. Pour cela, l'attraction doit se construire dans le sens du développement culturel, en permettant aux gens d'aller plus loin dans leur réflexion sur ce qu'ils ont et sur ce qu'ils sont capables de faire. Le cas des outils (murales, exhibits de plein air, etc.) inventés par la Haute-Beauce est révélateur de l'importance du médium muséographique dans la valorisation des rapports entre le visiteur et le visité. III s'agit de l'application concrète et planifiée, tant dans l'espace que dans le temps; de principes énoncés dans la Déclaration québécoise sur le tourisme culturel en 1979 .

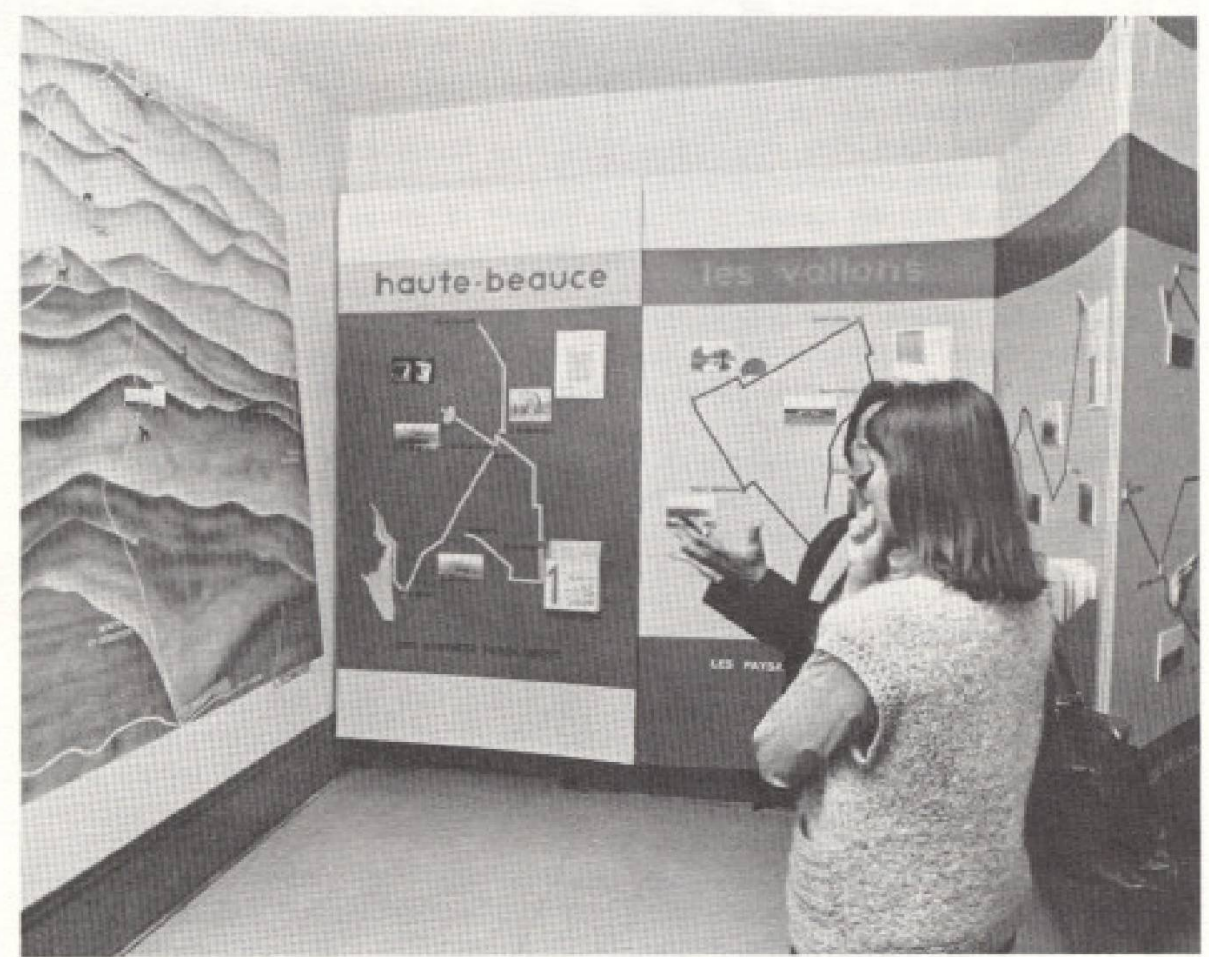

Dans le cadre de l'écomusée de la Haute-Beauce, une salle d'exposition qui révảle l'importance du médium muséegraphique dans la valorisation des rapports entre le visiteur et le visité. 
L'attraction est pour la région une promotion énorme sur le plan social où les objectifs sont strictement locaux; si le produit final permet d'attirer le touriste, de le retenir, et de crêer une image nationale intéressante, alors tant mieux. Ce travail, à l'origine, a fait en sorte que la population, repliée sur elle-même, très sédentaire, où l'exode est important, est sortie davantage à l'extérieur pour chercher de nouvelles idées. Elle est devenue elle-même, d'une certaine façon, touriste. La Beauce a vécu longtemps en vase clos. C'était sa force mais elle était statique et on la gardait ainsi en considérant qu'elle était la patrie du folklore. Les vieilles élites de la Beauce n'ont rien fait pour empécher que ce soit différent; c'est pour cela qu'il n'y a pas eu de développement culturel. On a conservé l'image ancienne comme un trésor, comme font les musées: la population fut considérée comme un musée.

Aujourd'hui, il faut se greffer sur ce filon fort et original mais également aller chercher des éléments extérieurs de culture qui vont régénérer la population et apporter des dêveloppements insoupçonnés. L'écomusée n'a pas pour objet de amuséifiers une population, de garder les vieilles choses. C'est tout a fait le contraire. Le territoire, sa population et son environnement deviennent l'objet de la valorisation - si objet il y a - au sens de témoins d'une culture. L'écomusée devient prétexte par rapport à la démarche globale d'appropriation de la vie culturelle et de ses modes d'expression.

En tourisme, on aime souvent les vieilles choses qu'on considère comme plus authentiques mais aussi parce qu'elles rassurent. Si on essaie de congeler les cultures pour en faire des exhibits à montrer aux touristes, on donne au passé un sens qu'il n'a pas en lui-meme. Les populations locales ont besoin de points de référence au passé mais c'est pour s'engager dans l'avenir, pour s'organiser sans perdre toute continuité. Elles ont déjà su faire les choses; elles doivent devenir les maîtres-d'ceuvres de leur développement. L'écomusée vise a la qualification professionnelle et culturelle des populations considérées sur le plan collectif.

\section{Une culture à définir}

La recherche systématique sur la question du Québec, l'identité culturelle, est récente et à peine annoncée. Lorsqu'on crée un musée de la civilisation, on doit produire de façon extrêmement rapide, comptetenu des impératifs budgétaires et politiques, alors qu'il faudrait une vingtaine d'années pour échelonner un programme comme celui-là pour qu'il arrive à une vision assez juste. Nous avons beaucoup de difficultés à définir notre identité na- tionale, à nous entendre comme peuple sur la culture. Nous sommes divisés politiquement, mais aussi sur le plan géographique, dans nos options de vie, dans nos convictions.

Si on ne se concerte pas comme peuple, alors essayons de le faire par le détour des régions. Mais on les connaît très peu finalement ces régions et elles ne participent pas à leur définition. Les préjugés dominent et on voit les régions à travers plusieurs prismes. Le lac St-Jean s'est imposé comme nationaliste. Dans le Bas du fleuve et la Gaspésie, on a fait du brassage artificiel dans l'espoir de voir ressortir certaines caractéristiques. Mais ces populations ont réalisé que ces caractéristiques ne correspondaient pas à leurs idées: elles se sont rebellées et repliées et commencent maintenant à se redéfinir. D'autres régions sont encore totalement inconnues, comme les Cantons de l"Est, où s'ajoute un problème ethnique; on fait alors beaucoup de simplifications:12). Bref, on parle de régionalisme et de nationalisme au lieu de parler de région et de mouvement national.

\section{La culture urbaine}

La culture urbaine, comme les cultures régionales, est importante mais très peu exploitée ${ }^{(13)}$. Dans le Centre-sud de Montréal, par exemple, le projet de l'écomusée de la Maison du Fier Monde est né comme un mouvement d'appoint à une autre démarche, celle des habitations communautaires. Les centres communautaires avaient des ressources pour réaliser des projets. Ils ont proposé que les gens du quartier retrouvent, à travers leur histoire, une volonté d'agir pour rebatit leur quartier. Le mouvement s'est défini comme w/e miroir de la population du Centre-Sud de même qu'un pole d'attraction important des forces vives du quartier. ${ }^{114^{\circ}}$ II en va de même du mouvement qui se dessine dans le quartier voi$\sin$ d'Hochelaga-Maisonneuve: I'Atelier d'Histoire et le projet de création d'un centre d'interprétation du développement industriel. Ce projet de meme que celui d'un centre d'interprétation de la vie collective et de la technologie ouvrière dans le Centre-sud visent a la ré-appropriation de leur culture par les classes ouvrières absentes du développement culturel urbain. Ils agissent dans cette perspective en complémentaritế indispensable au Centre d'interprétation de I'histoire de la Ville de Montréal - initiative du Ministère des Affaires culturelles et de la Ville de Montréal - qui ouvrira ses portes en juin 1983.

Les gens de ces quartiers ont intérêt a a faire reconnaittre le travail qu'ils font, tout en agissant sur leur milieu. II me semble important aussi d'offrir un produit que les gens de l'extérieur, y compris les touris- tes, puissent apprécier. Autrefois, on venait au Québec pour la vie champêtre, idéalisée bien souvent. On pourra maintenant offrir des excursions dans des quartiers de Montréal qui expriment de diverses façons notre culture urbaine. Pour cela, les populations des quartiers ouvriers devront créer. Ce qui identifie un mouvement populaire, ce $n^{\prime}$ est pas des équipements de moindre qualité, à rabais, c'est la prise en charge, un mode de gestion et de décision appartenant à la population. Ces mouvements apprennent à utiliser et à contrôler des équipements hautement techniques pour réaliser leurs objectifs.

Les centres d'interprétation de la culture urbaine, comme ceux de d'autres régions vont se donner des équipements culturels propres, de qualité et d'originalité comparables â d'autres moyens utilisés ailleurs. En réalisant qu'elles peuvent faire cela, les populations des régions et des quartiers urbains s'ouvrent la porte pour accomplir toutes sortes d'autres choses, I'objectif de la «population qualifiéew étant atteint.

Les visiteurs de passage, les touristes, retrouveront dans les centres d'interprétation régionaux et locaux de la culture québécoise, un produit complet: les gens, leurs ceuvres, leur histoire et leur milieu. S'ils ne peuvent parcourir tous les réseaux pour avoir une idée d'ensemble de la diversité des composantes de la culture québécoise, du moins prendront-ils contacts avec des micro-milieux culturels authentiques et vivants. C'est quand même mieux que de se laisser attraper par un miroir à alouettes! +

\section{Références}

11) Politique quabecoise du daveloppement culturel Vol. I, M.A.C. Québec, 1978, p. 47 .

(2) Voir a ce sujet, Le Tourismes aspeets thetoriquas at pratiquẹs au Qubbec, Sodilis, Montréal 1982, et notamment les chapitres sur roffre touristique de Roger NA DEAU et aur la demande touristique de Mare LAPLANTE. ainsi que leur article dans la présent numéro.

(3) Politique québdecise du dd́veloppement culturel, op. cit., vol, 1, p. 109.

(4) SEGUIN, R.L.: La eivilisation traditionnello do I'habitant, Montríal, Fides, 1973 , p. 96.

(5) Politique queblecoise... op. cit., vol. 1, p. 9 .

(6) Idem, p. 95 .

(7) Idem, p. 101,

(a) Idem, vol. II, p. 191

(9) BOULIzoN, Guy: Les mustes du Qubbec, Montrital, Fides, 1, 1976, p. 10.

(10) Le document: Les mushes du Qúbbec an devenir: concept musbologique. Ministêré des Affaires culturelles, 1979, propose un systemene comprohensif de la connais sance de Thomme of ici.

(11) Politique québłcoise..., op. eit., vol. 1, p. 53.

(12) C.f. le 10e rappoont de la Commission des biens culturels, $1981-82$.

(13) Montríal est confirmé dans sa wocation de mitropol culturelle. Voir: Des actions culturelles pour aujour d'hui, programme daction du Ministere des Affaires cultureiless, mai 1903 . La portice nationale de la culture urbaine se précisera dans les anndes a wenir.

(14) Masson du Fier-Monde, document de présentation, 1961, awant-propos. 